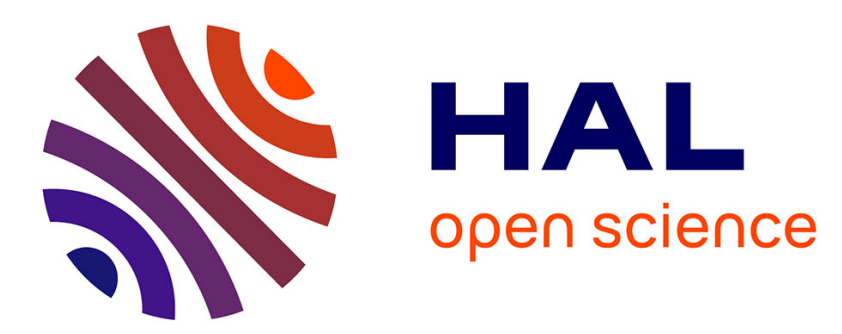

\title{
Uso del congiuntivo in italiano e francese in un corpus di fumetti e graphic novel
}

Sophie Saffi

\section{To cite this version:}

Sophie Saffi. Uso del congiuntivo in italiano e francese in un corpus di fumetti e graphic novel. Studia Universitatis Babeş-Bolyai Philologia, 2020, 65 (3), pp.225-245. 10.24193/subbphilo.2020.3.17 . hal03172059

\section{HAL Id: hal-03172059 \\ https://hal-amu.archives-ouvertes.fr/hal-03172059}

Submitted on 17 Mar 2021

HAL is a multi-disciplinary open access archive for the deposit and dissemination of scientific research documents, whether they are published or not. The documents may come from teaching and research institutions in France or abroad, or from public or private research centers.
L'archive ouverte pluridisciplinaire HAL, est destinée au dépôt et à la diffusion de documents scientifiques de niveau recherche, publiés ou non, émanant des établissements d'enseignement et de recherche français ou étrangers, des laboratoires publics ou privés. 


\title{
USO DEL CONGIUNTIVO IN ITALIANO E FRANCESE IN UN CORPUS DI FUMETTI E GRAPHIC NOVEL
}

\author{
SOPHIE SAFFI ${ }^{1}$
}

\begin{abstract}
Uses of the subjunctive in Italian and French in a corpus of comics and graphic novels. We propose the study of the uses of the subjunctive in Italian and French and the alternative solutions in each of the two languages on the basis of a corpus of 18 comics and graphic novels ( 9 originals and their translation) published from 1997 to 2017. 49,7\% of the 227 items obtained correspond to the use of the subjunctive in Italian and alternative solutions in French; 29\% to the use of the subjunctive in both languages; $21 \%$ to the use of the subjunctive in French and alternative solutions in Italian. We describe the profile of the uses, the syntactic context and the type of semantics involved. Our objective is to determine the systemic coherence of these uses and to produce an inventory of the use of the subjunctive in the French and Italian neostandards, as close as possible to the current use of the two languages, for the first two decades of the 21st century.
\end{abstract}

Keywords: subjunctive, French, Italian, comics

REZUMAT. Întrebuințarea conjunctivului în italiană și franceză într-un corpus de bandă desenată și de roman graphic. Pe baza unui corpus constituit din 18 benzi desenate și romane grafice ( 9 originale și traduceri) publicate în perioada 1997 - 2017, vom studia în articolul nostru utilizarea conjunctivului în italiană și în franceză, precum și soluțiile alternative în fiecare dintre cele două limbi. În ansamblul corpusului, 49,7\% din 227 intrări corespund utilizării conjunctivului în italiană și soluțiilor alternative în franceză; $29 \%$ trimit la utilizarea conjunctivului în ambele limbi; $21 \%$ reflectă utilizarea conjunctivului în franceză și soluții alternative în italiană. Descriem profilul de utilizare, contextul sintactic și tipul de semantică implicat. Obiectivul nostru este cel de a determina consistența sistemică a acestor utilizări, dorim totodată să furnizăm un inventar al acestora în nivelul neo-standard al limbii franceze și al celei italiene, apropiindune cât mai mult posibil de utilizarea contemporană a acestora, mai ales în primele decenii ale secolului XXI.

Cuvinte cheie : conjunctiv, franceză, italiană, benzi desenate 
Ci si propone lo studio degli usi del congiuntivo in italiano e in francese e delle soluzioni alternative in ciascuna delle due lingue sulla base di un corpus di 18 fumetti e Graphic Novel ${ }^{2}$ (per un totale di 108.977 parole) composto da 9 opere originali, 5 in francese (Golden city ; L'Appel; Quatuor ; Peplum ; Pauvres Zhéros) e 4 in italiano (Intervista a Pasolini ; Per questo mi chiamo Giovanni; Brancaccio ; Peppino Impastato), e le loro traduzioni in italiano o francese. Questi testi sono stati pubblicati dal 1997 al 2017. È stato selezionato un corpus eterogeneo sia per lo stile di scrittura che per i temi trattati al fine di ottenere un panel più rappresentativo possibile del mezzo fumettistico. L'unico vincolo era l'esistenza di una traduzione nell'altra lingua.

Il corpus comprende gli originali scritti in una lingua che rispetta lo standard, ma alcuni autori si permettono deviazioni dallo standard scritto per rappresentare meglio i dialoghi orali. Tuttavia, queste deviazioni rimangono nel quadro di ciò che è già generalmente accettato, per esempio l'omissione della ne di negazione prima del verbo in francese. I fumetti appartengono al genere delle Scritture brevi ${ }^{3}$, ma si differenziano dagli SMS ed e-mail perché sono una creazione artistica. Quindi offrono al linguista un'opera scritta non spontanea, di cui uno degli obiettivi è quello di proporre una ricostruzione del contesto del dialogo interlocutorio naturale e spontaneo: quella del suo autore.

Il nostro corpus combina gli originali e le loro traduzioni, che riflettono anche la lingua standard accettata. L'interesse di un insieme di testi misti è quello di permetterci di proporre ipotesi sull'uso del congiuntivo, che si basano su scritti in lingua standard lasciando spazio alle creazioni del neo-standard dell'orale più condiviso dalla comunità di madrelingua.

2 Il genere Fumetti-Graphic Novel presenta la caratteristica principale di un'arte del racconto per immagini come il cinema, e quindi di avere un legame tra testo e immagine. Il genere si specializza con una standardizzazione di soggetti e forme e l'apparizione, almeno nel mondo francofono, di una cultura Fumetto negli anni Ottanta, quando la cultura underground diventa dominante. La Graphic Novel è una categoria nata dalla porosità tra fumetto e letteratura, e dalle iniziative di autori underground degli anni ' 60 e '70, le cui produzioni hanno in comune la caratteristica di non cercare un bel disegno, di rivolgersi a un lettore adulto e di affrontare temi difficili. Il Graphic Novel italiano rivendica lo statuto di movimento letterario con una strategia di diffusione negli scaffali letterari delle librerie adottando un formato diverso dal fumetto e più vicino a quello del romanzo.

${ }^{3}$ Le Short Writings o Scritture brevi si definiscono dal punto di vista della variazione diatecnica, cioè l'aspetto della variazione che dipende dai diversi mezzi tecnici utilizzati come strumenti di comunicazione linguistica. Questi testi brevi appartengono a specifici generi testuali (ad es. fumetti, tweet, chat online) che presentano una complessità di bi-polarizzazione diametica tradizionale, così come alcune varietà di testi antichi e moderni (come iscrizioni su epigrafi, tavolette di piombo, monete, ecc.) che possono essere rilevati in testi scritti anche se tradizionalmente attribuiti alla parola $\mathrm{o}$, al contrario, possono essere rilevati nella lingua parlata anche se tradizionalmente attribuiti a testi scritti. Chiusaroli (2012a: 7) nota per gli SMS, e Saffi (2016: 427) per i fumetti, che questi testi sono caratterizzati da una rinnovata modalità espressiva grafica che in qualche modo annulla la distinzione dei tradizionali concetti di variazione diametica tra scritto e parlato. 
Il nostro obiettivo è quello di determinare la coerenza sistemica di questi usi e di farne l'inventario nei neo-standard francese e italiano, il più vicino possibile all'uso attuale delle due lingue, per i primi due decenni del XXI secolo.

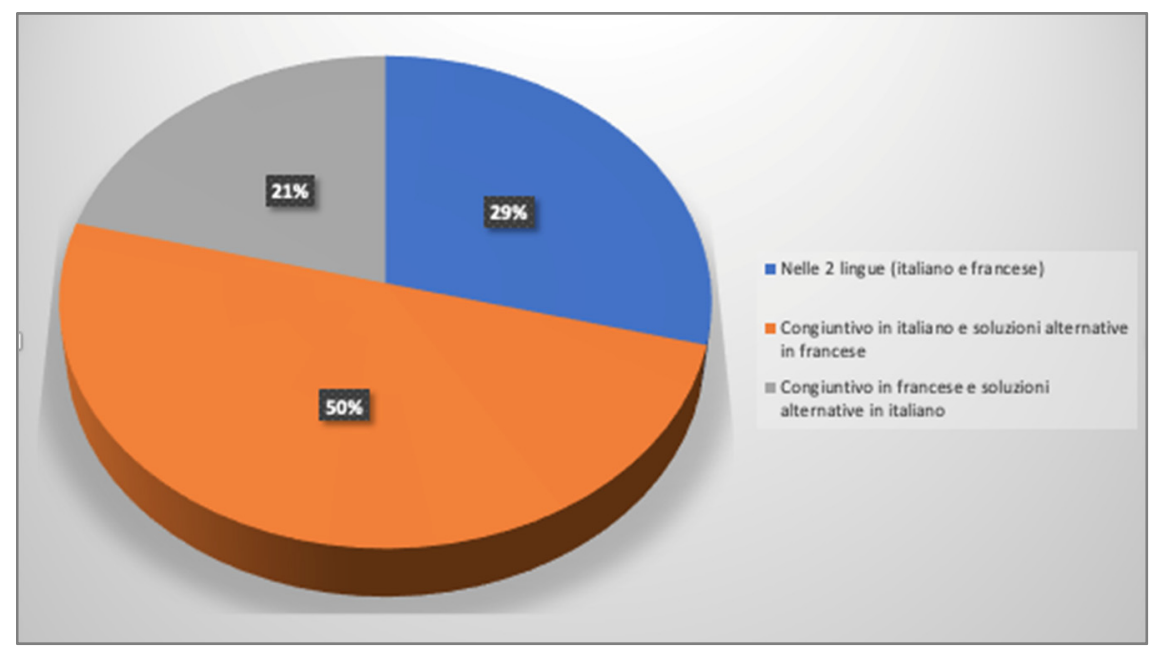

Fig. 1. Uso del congiuntivo in un corpus di fumetti italiani e francesi

I risultati del corpus sono suddivisi in 3 categorie:

1) Gli usi del congiuntivo in italiano corrispondenti a soluzioni alternative in francese rappresentano la soluzione maggioritaria: il 49,7\% dei casi (113 voci su un totale di 227).

2) Gli usi del congiuntivo in italiano e francese rappresentano il $29 \%$ delle occorrenze (66 su un totale di 227).

3) Gli usi del condizionale in francese corrispondenti a soluzioni alternative in italiano rappresentano il $21,1 \%$ dei casi (48 voci su un totale di 227 ).

Così, gli usi del congiuntivo sono più numerosi nel corpus italiano che in quello francese, come ci si poteva aspettare sulla base dei risultati delle grammatiche descrittive dell'italiano standard (Dardano: 2009; Tekavčić: 1972). Il francese contemporaneo, invece, non applica strettamente la concordanza dei tempi osservata nel sistema italiano ed ereditata dalla consecutio temporum latina (Brunot, 1965: 782; Begioni \& Rocchetti, 2013).

La concordanza dei tempi risponde all'esigenza di coerenza nella frase complessa, il che si ottiene facendo combaciare l'aspetto formale, modale e quello temporale dei sintagmi verbali della proposizione reggente e della sua o delle sue subordinate. Così, in latino, il verbo della subordinata riprende i segni 
aspettuali, temporali e modali del verbo della reggente. Tuttavia, con il movimento evolutivo di deflessione dal latino alle lingue romanze, questa necessità è sempre meno sentita, e finisce per essere vista come una ridondanza quando il legame sintattico tra le proposizioni si stabilisce per mezzo di congiunzioni di subordinazione (fr. Je pense que c'est vrai vs. it. Penso (che) sia vero). Il movimento di deflessione verbale dal latino alle lingue romanze contemporanee è passato attraverso tappe intermedie. In italiano e in francese classico, il verbo subordinato riprende solo i segni del tempo e del modo (it. voglio (che) venga / volevo (che) venisse; fr. class. je veux qu'il vienne / je voulais qu'il vînt [Begioni \& Rocchetti, 2013 : 34]), in francese contemporaneo, si constata in certi casi la ripresa dei soli segni modali (fr. Dans ce cas, il vaudrait peut-être mieux que je prenne les commandes, vous ne croyez pas? [Goldy-fr-cond-5-b] vs. it. In questo caso forse sarebbe meglio che prendessi io i comandi, non crede? [Goldy-it-cond-5-b]).

Begioni \& Rocchetti (2013: 27) ritengono che l'evoluzione che abolisce la necessità di assemblare le due forme verbali della reggente e della subordinata, che tende a riunire informazioni formali e semantiche e a focalizzarle nel verbo della reggente, termina quando non c'è più duplicazione di informazioni. Mettono in relazione "queste evoluzioni ad una ristrutturazione della frase dovuta al passaggio dall'indoeuropeo, lingua agglutinante, con il verbo in posizione finale, alle attuali lingue indoeuropee che integrano diverse proposizioni incorporate le une nelle altre, una o alcune delle quali sono reggenti e le altre subordinate" 4 . Così, essi tracciano un parallelo tra "il processo di creazione dell'articolo a partire dal pronome [che] risulta dall'applicazione della deflessione al sintagma nominale" e "la creazione della subordinazione [che] è l'applicazione dello stesso processo al sintagma verbale" ${ }^{5}$. Tracciano un parallelo tra i segni del genere e del numero nel sintagma nominale e i segni del modo e del tempo nei sintagmi verbali della reggente e della sua subordinata: secondo loro, "lo scopo della subordinazione è infatti la trasformazione di una frase nell'equivalente di un sostantivo in modo che il tutto così "sostanziato" possa essere integrato in un'altra frase"6 (Begioni \& Rocchetti, 2013: 34).

Il nostro corpus ci permette di descrivere l'evoluzione dei sistemi contemporanei italiano e francese in questo processo.

4 « [...] ces évolutions avec une restructuration de la phrase due au passage de l'indo-européen, langue agglutinante, avec le verbe en position finale, aux langues indo-européennes actuelles intégrant plusieurs propositions enchâssées les unes dans les autres, dont l'une ou les unes sont régissantes et les autres régies »

5 « [...] le processus de création de l'article à partir du pronom [qui] résulte de l'application de la déflexivité au syntagme nominal »; «la création de la subordination [qui] est l'application du même processus au syntagme verbal »

6 « [...] l'objectif de la subordination est en effet la transformation d'une phrase en l'équivalent d'un substantif pour que l'ensemble ainsi « substantivé » puisse être intégré dans une autre phrase » 


\section{Uso del congiuntivo nelle due lingue}

Si è constatato l'uso del congiuntivo in entrambe le lingue principalmente nelle subordinate: completive: $56 \%$, circostanziali: $16,6 \%$, relative: $7,5 \%$. Nel $15 \%$ dei casi si tratta di una proposizione clivée (cosiddetta falsa relativa) ${ }^{7}$. Sono stati trovati un solo caso di proposizione indipendente $(1,5 \%)$ e due casi di uso del congiuntivo nella costruzione di locuzioni (3\%; fr. quoi que ce soit, quelle que soit; it. qualsiasi, quale che sia la cui composizione include una relativa: pronome + relativa).

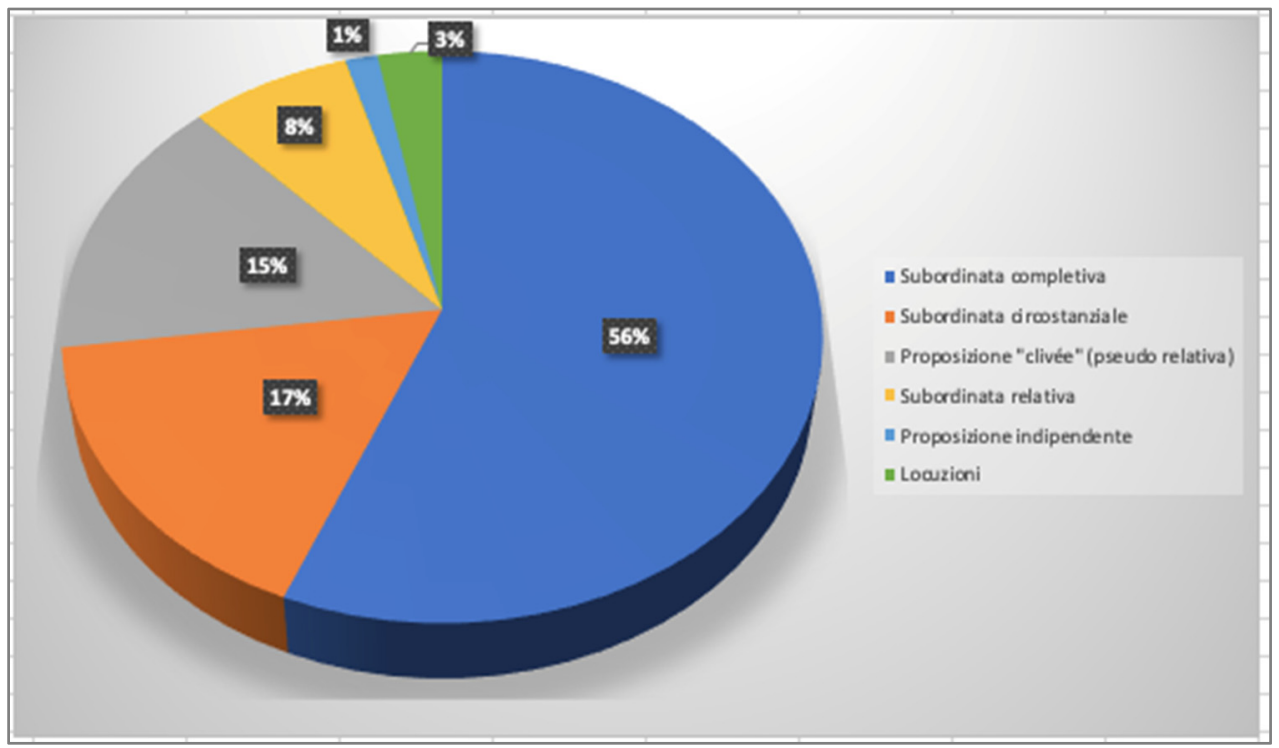

Fig. 2. Contesti d'uso del congiuntivo nelle due lingue

Il contesto maggioritario in cui il congiuntivo è usato in entrambe le lingue è quello di una subordinata congiuntiva completiva:

(Appel-cond-2) - Il faudra que j'aille commander de la toile aux camionneurs. (Appello-cond-2) - Bisognerà che ordini della tela ai camionisti

(Giov-fr-cond-24) -C'est ça. Giovanni ne veut pas qu'une autre fille coure ce danger. [...]

(Giov-it-cond-22) - Infatti. Giovanni non vuole che un'altra ragazza corra lo stesso pericolo. [...]

${ }^{7}$ Per i criteri di discriminazione tra relativa e clivée: cf. Brault-Scappini, 2007. Per una bibliografia sulle clivées: cf. Rouquier, 2018. 


\section{Ci si aspettava questo risultato sapendo che:}

« [...] i verbi francesi e italiani dipendono semanticamente dalla soggettività di chi parla, sia in forma personale (fr. vouloir, souhaiter, désirer..., it. volere, augurarsi, desiderare...) o impersonale (fr. il faut que, il est nécessaire que, il est possible que, il se peut que..., it. bisogna/occorre che, è necessario che, è possibile che, può darsi che...) [...] richiedono nella proposizione subordinata una modalità virtuale, resa sia in francese che in italiano dal congiuntivo. $»^{8}$ (Begioni \& Rocchetti, 2013: 34-35)

Poi, in ordine decrescente di frequenza, una subordinata congiuntiva circostanziale, con 6 item di subordinata di scopo, 2 item di subordinata di tempo, 1 item di subordinata di luogo, 1 item di concessiva e 1 item di subordinata di condizione:

Scopo:

(Goldy-fr-cond-29) - [...] J'ai été obligée de l'enfermer pour qu'elle n'agresse plus personne !...

(Goldy-it-cond-27) - [...] L'ho dovuta rinchiudere affinché non aggredisca più nessuno!...

Tempo:

(Paso-fr-cond-7) - ... J'aurai des choses à vous montrer avant que vous ne partiez. Pouvons-nous parler frioulan ? Vous parlez le frioulan?

(Paso-it-cond-7-b) - Mi piacerebbe farle vedere delle cose prima che se ne vada. Possiamo parlare in friulano? Lei parla friulano?

Tra queste 11 occorrenze, 2 presentano la particolarità di avere un contesto sintattico diverso in francese e in italiano:

Subordinata di scopo in francese, con la reggente sotto-intesa: risposta a una domanda:

(Goldy-fr-cond-10) - Pour que vous me disiez où sont les hydrojets.

Completiva in italiano: la risposta viene elusa e sostituita da un'affermazione:

(Goldy-it-cond-10) -Basta solo che mi diciate dove sono gli idrogetti.

Concessiva in francese:

(Paso-fr-cond-34-b) Environ un an après, et après qu'ils aient rencontré une quantité de femmes sans qu'aucune ne leur convienne à tous les deux...

8 «[...] les verbes français et italiens dépendant sémantiquement de la subjectivité du locuteur, qu'ils se présentent sous une forme personnelle (fr. " vouloir, souhaiter, désirer... », it. volere, augurarsi, desiderare...) ou impersonnelle (fr. " il faut que, il est nécessaire que, il est possible que, il se peut que... », it. bisogna/occorre che, è necessario che, è possibile che, può darsi che...) [...] requièrent dans la proposition subordonnée un mode virtuel, rendu en français comme en italien par le subjonctif. » 
Relativa in italiano:

(Paso-it-cond-42) Circa un anno dopo e dopo avere incontrato tante femmine e nessuna che potesse andare bene a tutti e due...

Questi esempi illustrano il ruolo del traduttore le cui scelte sintattiche influiscono sulla comprensione del lettore.

Nel 16,6\% dei casi, il congiuntivo è utilizzato in una clivée. Questo processo di messa a fuoco è ampiamente usato nel francese parlato e si trova anche nel codice scritto: la parte estratta che si vuole mettere a fuoco è introdotta da $c^{\prime}$ est; in alcuni casi è anche usato il $y a$. La parte estratta è seguita da una relativa introdotta da qui se il gruppo estratto è il soggetto della relativa, e da que in tutti gli altri casi. Nel nostro corpus, il verbo di questa relativa è coniugato al congiuntivo:

(Goldy-fr-cond-6-b) - [...] Pourtant c'est impossible que ce soit lui puisqu'il est actuellement au bagne !

(Goldy-it-cond-6-b) - [...] Eppure è impossibile che sia lui visto che si trova in prigione!

(Goldy-fr-cond-9) - C'est incroyable que vous ayez réussi à vous évader de là-bas ! ... Personne n’y était arrivé jusqu'à présent !

(Goldy-it-cond-9) - È incredibile che sia riuscito a evadere da quel posto! Nessuno fino ad oggi c'era mai riuscito!

(Giov-fr-cond-42) -Regarde là-haut... Il est juste que tous ceux qui débarquent à Palerme sachent immédiatement que ce n'est pas la ville de la mafia : c'est la ville de Giovanni et de Paolo.

(Giov-it-cond-39) - Leggi là sopra... È giusto che chi arriva a Palermo sappia subito che questa non è la città della mafia: questa è la città di Giovanni e di Paolo.

(Quatuor_cond_5) - C'est un MIRACLE que je vous aie gardé si longtemps !

(Quartetto_cond_5) - Ė un miracolo che non l'abbia licenziata prima !

Sono anche stati rilevati delle occorrenze di proposizioni relativi (15\%):

(Giov-it-cond-38-a) - Giovanni aveva rinunciato ad avere un figlio, cioè alla gioia più grande che si possa provare, perché sapeva che un giorno la mafia l'avrebbe ucciso.

(Giov-fr-cond-41-a) Giovanni avait renoncé à avoir des enfants, c'est-àdire à la plus grande joie qui soit, parce qu'il savait qu'un jour, la mafia le tuerait.

(Goldy-fr-cond-1) - C'est le plus beau cadeau qu'on m'ait jamais fait !

(Goldy-it-cond-1) - Ė il più bel regalo che abbia mai ricevuto!

Se si confrontano con i contesti d'uso del congiuntivo in italiano quando il francese usa una soluzione alternativa, e viceversa (congiuntivo in francese, soluzione alternativa in italiano), si constata che: 
- le subordinate completive (compresi le interrogative indirette e le completive con funzione soggettiva in italiano) rappresentano il contesto maggioritario d'uso del congiuntivo, tranne nel caso d'uso del congiuntivo in italiano con una soluzione alternativa in francese, in cui le subordinate circostanziali sono di poco il contesto maggioritario (completive $42,4 \%$, circostanziali 44,2\%).

- L'italiano ha la caratteristica di utilizzare regolarmente il congiuntivo in una subordinata di condizione, cosa che non avviene in francese, dove la forma del congiuntivo non è stata mantenuta dopo la particella di subordinazione si:

«Infatti, que pone mentre si suppone. Il congiuntivo è quindi necessario dopo que, per esprimere la virtualità quando è richiesta dal verbo della reggente. Alle stesse condizioni, l'indicativo è richiesto dopo la congiunzione ipotetica si. $»^{9}$ (Begioni \& Rocchetti, $2013: 30$ )

- Le subordinate relative (comprese le clivées in francese e le locuzioni di tipo fr. Quoi qu'il en soit, quoi que ce soit, quelle que soit et it. qualsiasi, quale che sia in quanto, come sopra indicato, queste locuzioni incorporano una relativa nella loro composizione) competono con le subordinate circostanziali per il secondo posto nella classifica dei contesti d'uso del congiuntivo.

\begin{tabular}{|c|c|c|c|}
\hline \multirow[t]{2}{*}{ Contesto sintattico } & \multicolumn{3}{|c|}{ Uso del congiuntivo } \\
\hline & nelle due lingue & $\begin{array}{l}\text { in italiano e } \\
\text { soluzioni alternative } \\
\text { in francese }\end{array}$ & $\begin{array}{l}\text { in francese e } \\
\text { soluzioni alternative } \\
\text { in italiano }\end{array}$ \\
\hline Subordinata completiva & $56 \%$ & $42,4 \%$ & $60,4 \%$ \\
\hline Subordinata circostanziale & $16,6 \%$ & $44,2 \%$ & $20,8 \%$ \\
\hline di condizione & $1,5 \%$ & $41,5 \%$ & - \\
\hline di concessione & $1,5 \%$ & $1,7 \%$ & $4,1 \%$ \\
\hline di scopo & $9 \%$ & $0,8 \%$ & $10,4 \%$ \\
\hline di tempo & $3 \%$ & - & $6,2 \%$ \\
\hline $\begin{array}{ll}\text { di luogo } \\
\end{array}$ & $1,5 \%$ & - & - \\
\hline Costruzione di tipo 'relativa' & $25,5 \%$ & $8,8 \%$ & $16,6 \%$ \\
\hline Proposizione "clivée" & $15 \%$ & - & - \\
\hline Subordinata relativa & $7,5 \%$ & $7,9 \%$ & $10,4 \%$ \\
\hline Locuzioni & $3 \%$ & $0,8 \%$ & $6,2 \%$ \\
\hline Proposizione reggente & - & - & $2 \%$ \\
\hline Proposizione indipendente & $1,5 \%$ & $4,4 \%$ & - \\
\hline
\end{tabular}

Fig. 3. Confronto dei contesti sintattici d'uso del congiuntivo in italiano e francese in un corpus di fumetti

${ }^{9}$ « En effet, que pose alors que si suppose. Le subjonctif est donc nécessaire, après que, pour exprimer la virtualité lorsque celle-ci est exigée par le verbe de la principale. Dans les mêmes conditions, l'indicatif s'impose après la conjonction hypothétique si. » 


\section{Uso del congiuntivo in italiano corrispondente a soluzioni alternative in francese}

Si tratta della soluzione maggioritaria rilevata nel corpus (50\%). Le soluzioni alternative in francese all'utilizzo del congiuntivo italiano sono in ordine decrescente di frequenza:

\subsection{Indicativo imperfetto o più che perfetto: $42 \%$}

(Giov-fr-218) -Tu sais ce qui se passerait si Tonio faisait régner sa loi pendant plus d'un siècle et qu'il continuait à voler l'argent

(Giov-it-cond-7-b) -Sai cosa succederebbe se Tonio per un secolo intero continuasse a intascare i soldi dei compagni di classe?

(Pauvres_Zheros_cond_11) - ... Il a rien vu, et même s'il avait vu quelque chose, faudrait pas trop s'y fier, non?

(Povere_nullità_cond_12) - ... Non ha visto niente, e anche se avesse visto qualcosa c'è mica da fidarsi troppo, no?

Si è già indicato che l'italiano usa il congiuntivo in una subordinata di condizione mentre il francese usa l'indicativo in questo caso. Infatti, la virtualità delle subordinate di condizione è, in francese, portata dalla particella di subordinazione, mentre in italiano è condivisa tra quest'ultima e la modalità congiuntiva del verbo. In generale, le congiunzioni di subordinazione possono essere facoltative in italiano, mentre sono indispensabili in francese.

\subsection{Indicativo presente o passato prossimo: $37 \%$}

(Mafia_tabloids-201) - En effet, on peut penser que des génies ont construit ces rues complexes!

(Peppino_cond_5-b) - In effetti, si potrebbe pensare che siano stati dei geni a inventare quelle strade arzigogolate!

(Quatuor-fr-212) - Elle est belle la future princesse, vous ne trouvez pas?

(Quartetto_cond_26) - Non trova che la futura principessa sia bella?

Il primo esempio illustra il fatto che in francese in generale l'aspetto semantico del verbo della reggente (in questo caso: fr. penser vs. it. pensare) gioca un ruolo più decisivo che in italiano nella semantesi dell'intera frase. L'opposizione aspettuale perfettivo/imperfettivo è un'opposizione semantica in francese, si basa sul significato del verbo e non è resa né per coniugazione né per un qualsiasi affisso sistematico. Wilmet (1997: § 386) specifica che "una prima decantazione avviene tra l'aspetto semantico e l'aspetto formale"10 secondo

10 « une première décantation s'opère entre l'aspect sémantique et l'aspect formel » 
il significato del verbo o un indizio concreto che trasmette l'informazione aspettuale. La stessa osservazione vale per il sintagma verbale della reggente nel primo esempio (fr. on peut penser, it. si potrebbe pensare): in francese, la semantica del sintagma verbale è sufficiente a evocare la possibilità di un'opinione e tutta la frase è coniugata all'indicativo, mentre in italiano questo ruolo è svolto dall'uso del condizionale nella reggente e del congiuntivo nella subordinata.

Nel secondo esempio, l'inversione della costruzione sintattica tra francese e italiano spiega il cambio di modo: in francese, il locutore inizia con un'affermazione (indicativo) e poi chiede all'interlocutore il suo parere; in italiano, il locutore chiede direttamente il parere dell'interlocutore (congiuntivo). La paratassi usata in francese è attribuibile al genere Fumetto, perché questa figura stilistica del discorso copia la lingua parlata e rende la spontaneità dei dialoghi. Tuttavia, la versione italiana dello stesso fumetto presenta un'ipotassi. Questa preferenza va di pari passo con la regolarità della concordanza dei tempi in italiano: è sistemica.

\subsection{Condizionale: $7 \%$}

(Giov-fr-cond-31) Leoluca, par exemple, sans jamais nommer Giovanni, laisse entendre que celui-ci serait du côté du monstre...

(Giov-it-cond-29) Leoluca, per esempio, non fa il nome di Giovanni, ma sembra suggerire che stia della parte del mostro...

(Paso-fr-cond-24) Je pense que Pasolini, quand il était jeune et qu'il fouillait la librairie, aurait pu imaginer cette possibilité...

(Paso-it-cond-30) Quando Pasolini era ragazzo e cercava dentro la libreria penso avesse immaginato questa possibilità...

In questi esempi, l'uso del condizionale aiuta ad attenuare il significato dell'affermazione ed è sintomatico del fatto che il locutore non assume la responsabilità dell'affermazione (Abouda, 2001). Se il condizionale è sostituito da un altro tempo dell'indicativo, l'enunciato diventa molto più assertivo (Leoluca laisse entendre que Giovanni serait du côté du monstre vs. Leoluca laisse entendre que Giovanni est / était / sera du côté du monstre; Je pense que Pasolini aurait pu imaginer cette possibilité vs. Je pense que Pasolini a pu / avait pu / pourra imaginer cette possibilite).

Si potrebbe pensare che il ruolo del verbo servile pouvoir non sia insignificante in francese, che fornisce informazioni sul carattere ipotetico della semantesi verbale, tratto che viene trasmesso in italiano attraverso l'uso del congiuntivo. Tuttavia, questa sarebbe una falsa interpretazione, poiché nel nostro corpus sono stati trovati casi d'uso anche in italiano di dovere e riuscire in quanto verbi servili coniugati al congiuntivo imperfetto e seguiti dai verbi 
tradirti e deciderti all'infinitivo, corrispondenti in francese dei verbi trahir e décider coniugati al condizionale:

(Quatuor_cond_36) - Dans le cas où ta mémoire te trahirait, tant pis pour ton tailleur...

(Quartetto_cond_43) - Nel caso in cui la tua memoria dovesse tradirti, puoi salutare il tuo sarto ...

(Quatuor_cond_40) - Pourquoi erres-tu comme une femme folle dans le jardin, remuant les lèvres comme si tu cherchais à dire quelque chose et tu ne te déciderais pas à le PRONONCER ?!?

(Quartetto_cond_46-b) - Perché vaghi per il giardino come una folle, muovendo le labbra come se cercassi di dire qualcosa e non riuscissi a deciderti di FARLO ?!

\subsection{Forma non verbale: $6 \%$}

(Bran-fr-201) -Tu as raison. Mais, hélas, leur présence ça plaît pas à certains, et ils viennent se plaindre à moi...

(Bran-it-cond-7) -E non hai ragione? Però purtroppo alla gente non piace che stiano lì, e poi vengono a lamentarsi con me...

(Pauvres_Zheros-201) - Mais ça aussi, c'est des conneries... Ma chienne, moi, elle renifle même sous la pluie...

(Povere_nullità_cond_9) - Ma anche queste sono tutte cazzate... La mia cagna, mi creda, riesce a fiutare anche sotto la pioggia...

\subsection{Infinitivo: $3 \%$}

(Giov-fr-202) -Peut-être qu'à une époque, quand l'Italie venait à peine de se former, on avait effectivement besoin de groupes pour défendre les plus faibles. Quand l'État ne disposait pas encore de solides institutions. (Giov-it-cond-13) - Forse un tempo, quando l'Italia era appena nata, c'era davvero bisogno di associazioni che difendessero i più deboli, perché lo stato non aveva ancora istituzioni salde.

\subsection{Futuro: $2 \%$}

(Paso-fr-206) - Parce que je ne sais pas dire non, parce que je me dis que ça servira à l'interviewer et à quiconque écoutera, etc., etc.

(Paso-it-cond-8) - Perché non so dir di no, perché penso che sia utile all'intervistatore e a chi ascolta, ecc. ecc.

(Paso-fr-205) J'espère qu'il m'écrira bientôt... Qu'il n'a pas réellement fait ce projet insensé.

(Paso-it-cond-67-a) Spero che mi scriva presto... che non sia davvero questo il suo folle progetto. 
Quest'ultimo esempio permette di distinguere la scelta tra futuro e passato prossimo in francese quando l'italiano usa in entrambi i casi il presente del congiuntivo: in italiano prevale l'informazione modale mentre il francese usa i tempi indicativi per la loro informazione temporale, essendo la caratteristica ipotetica dei processi già data dalla semantica del verbo della reggente (J'espère).

\subsection{Imperativo: $2 \%$}

(Goldy-fr-201) - Oui ! ... Mais à une condition : promets-moi de recommencer à manger, d'accord?

(Goldy-it-cond-13) - Sì!... Ma a condizione che tu mi prometta di ricominciare a mangiare, d'accordo?

La costruzione sintattica italiana (locuzione congiuntiva a condizione che + congiuntivo) corrisponde in francese a una successione di proposizioni indipendenti il cui legame è reso nello scritto dal ":" ma che, nella parola parlata, non richiede alcuno strumento particolare per rafforzare la coerenza tra la semantesi di queste proposizioni. La stessa osservazione vale in generale per un confronto tra il discorso italiano e quello francese: l'italiano utilizza spesso connettori come quindi, eppure, oppure, tuttavia, purtroppo, ecc. non utilizzati in francese.

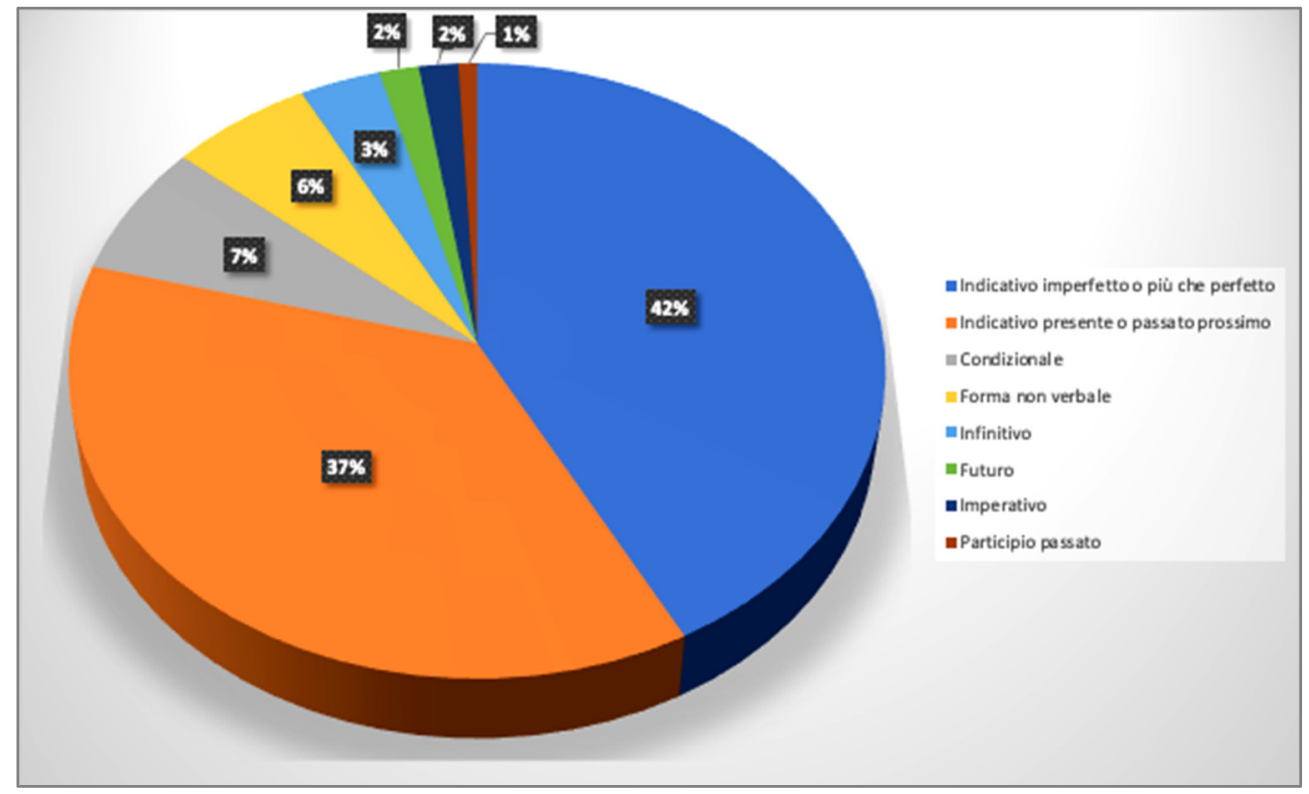

Fig. 4. Soluzioni alternative in francese all'uso del congiuntivo in italiano 


\subsection{Participio passato: $1 \%$}

(Giov-fr-201) C'est une rafale de "coupables », comme sortis du canon d'une kalachnikov, six cents « coupables » par minute.

(Giov-it-cond-26) Una raffica di 'condannato' come se li sparasse col kalashnikov, seicento 'condannato' al minuto.

\section{Uso del congiuntivo in francese corrispondente a soluzioni alternative in italiano}

Il congiuntivo è meno usato in francese che in italiano, quindi questa categoria è quella meno nutrita (20\%). Le soluzioni alternative in italiano

all'uso del congiuntivo in francese sono in ordine decrescente di frequenza:

\subsection{Indicativo presente: $32 \%$}

(Paso-it-311) [...], oppure ho solo bisogno vedere ancora una volta l'opera di Paul Mc Carthy, proprio davanti alla fontana.

(Paso-fr-cond-1) Il se peut aussi que j'aie simplement besoin de voir, une fois encore, l'œuvre de Paul Mc Carthy.

(Povere_nullità-307 et 306) - ... E poi bussano appedate contro la porta e poi cercano di aprirla!!

(Pauvres_Zhéros_cond_2-a et b) - ... Et pis qu'ils tapent à coups de pied dans la porte, pis qu'ils essaient de l'ouvrir !!

\subsection{Infinitivo: $29 \%$}

(Bran-it-302) - Devo andare, Carme'... tanto ci vediamo dopo, no? (Bran-fr-cond-2) -Faut que j'y aille, Carmelo... Mais on se voit après, non? (Giov-it-304) La persona che adesso non c'è più si era sacrificata per noi, per non esserci più mafia, per farci vivere un futuro più bello.

(Giov-fr-cond-46) Cette personne qui n'est plus là s'est sacrifiée pour nous, pour que la Mafia disparaisse, pour nous offrir un avenir meilleur.

\subsection{Forma non verbale: $23 \%$}

(Giov-it-301) - Ma potrebbe anche succedere che tu spaventato, dia a Tonio i tuoi cinque euro, e come tutti gli altri.

(Giov-fr-cond-4-b) -Mais il se pourrait aussi que, mort de peur, tu donnes tes cinq euros à Tonio, et que tous les autres enfants en fassent autant. (Paso-it-304) - [...] prestarsi in qualche modo a contribuire a questa marcescenza è, ora, il fascismo. 
(Paso-fr-cond-45) - [...] le fascisme, aujourd'hui, revient à contribuer de quelque façon que ce soit à cette marcescence.

(Paso-it-303) - Vada verso Roma e aspetti un mio segnale

(Paso-fr-cond-37-a) - Dirigez-vous vers Rome et attendez que je vous fasse signe.

\subsection{Condizionale: $6 \%$}

(Goldy-it-cond-46-b) - Temevo che mi avresti condannata!... Ancor peggio, che mi avresti odiata!... Non l'avrei sopportato: sarei morta di dolore!... Così ho preferito tacere.

(Goldy-fr-cond-49-a) - Je redoutais que tu me condamnes !... Pire : que tu me haïsses !... Je ne l'aurais pas supporté : je serais morte de chagrin !... Aussi ai-je préféré me taire.

\subsection{Indicativo imperfetto o il più che perfetto: $4 \%$}

(Giov-it-305) - Andai lì per l'inaugurazione. Vicino alla statua avevano messo delle panchine di legno dove i ragazzi potevano sedersi, guardare Giovanni e pensare a lui.

(Giov-fr-cond-52) -J'y suis allée pour l'inauguration. Juste à côté de la statue, ils avaient même mis des bancs, pour que les jeunes puissent s'asseoir, regarder la statue et penser à Giovanni.

(Bran-it-303) - Com'è che ancora non gli avete fatto la tac?

(Bran-fr-cond-14) - Comment ça se fait que vous lui ayez pas encore fait un scanner?

\subsection{Futuro: $2 \%$}

(Quartetto-it-307) - E non mi pare di avere avuto molte difficoltà a ricordare il mio nome. Siete stato buono, signore, ma temo che tra un anno stringerete tra le braccia solo vento e rimpianto.

(Quatuor_cond_37-b) - Et je ne vois pas que j'aie eu beaucoup de difficultés à me souvenir de mon prénom. Vous avez été bon, Seigneur, mais dans un an, je crains que vous ne serriez dans vos bras que du vent et du regret.

\subsection{Imperativo: $2 \%$}

(Peplum-it-303) - AHAHAH! Non farti di queste illusioni.

(Peplum_vf_cond_13-b) - HAHAHA ! Je ne voudrais pas que tu aies de telles illusions. 


\subsection{Gerundio: $2 \%$}

(Peplum-it-302) - Salve a te, campagnolo. Che cosa brucia dietro la cima della montagna creando un tale pennacchio?

(Peplum_vf_cond_11) - Salut à toi, campagnard. Que fait-on brûler derrière ce piton qui produise un tel panache?

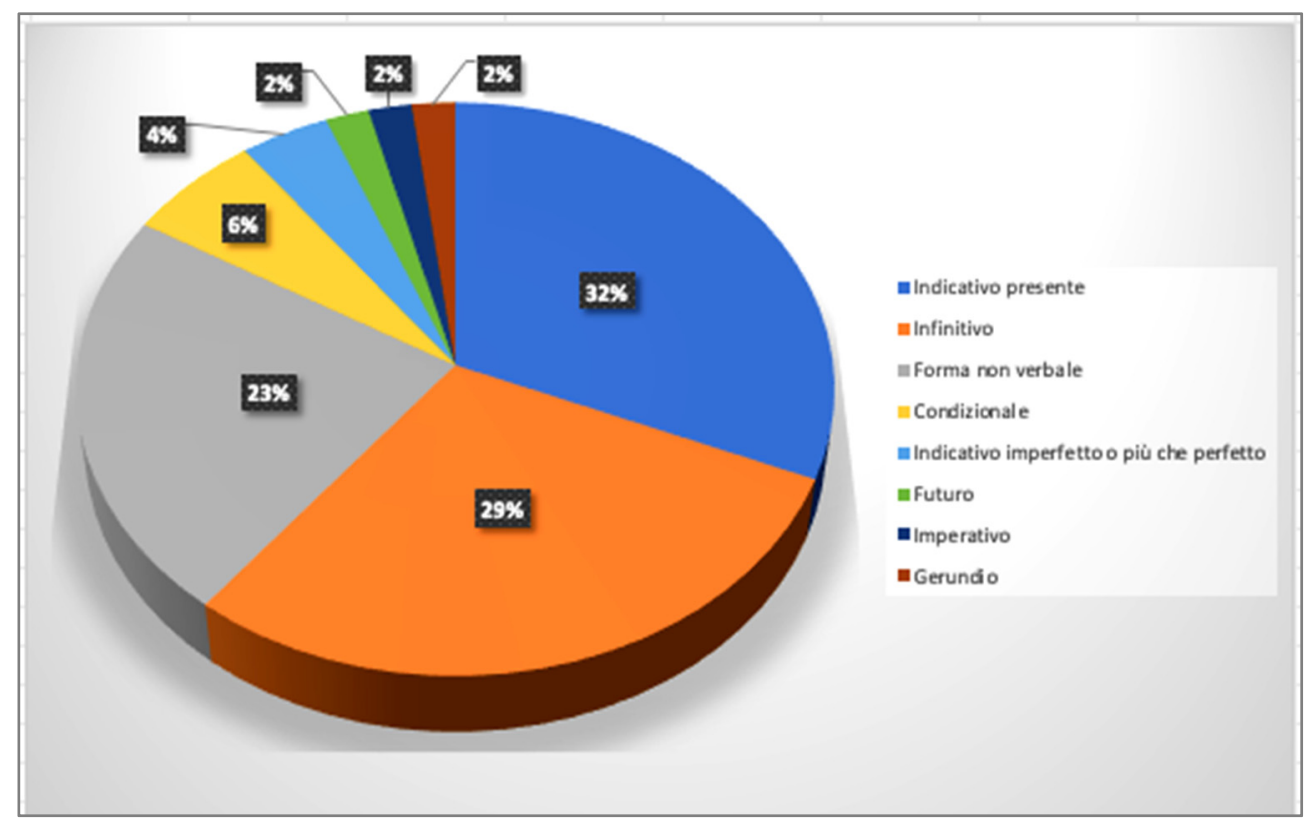

Fig. 5. Solutions alternatives en italien à l'emploi du subjonctif en français

4. Confronto francese-italiano delle soluzioni alternative all'uso del congiuntivo

\begin{tabular}{|l|c|c|}
\hline & Francese & Italiano \\
\hline Indicativo presente & $\mathbf{3 7} \%$ & $\mathbf{3 2} \%$ \\
\hline Indicativo imperfetto o più che perfetto & $42 \%$ & $4 \%$ \\
\hline Infinitivo & $3 \%$ & $29 \%$ \\
\hline Forma non verbale & $\mathbf{6 \%}$ & $23 \%$ \\
\hline Condizionale & $\mathbf{7 \%}$ & $\mathbf{6 \%}$ \\
\hline Futuro & $\mathbf{2 \%}$ & $\mathbf{2 \%}$ \\
\hline Imperativo & $\mathbf{2 \%}$ & $\mathbf{2 \%}$ \\
\hline Participio passato o gerundio & $\mathbf{1 \%}$ & $\mathbf{2 \%}$ \\
\hline
\end{tabular}

Fig. 6. Confronto francese-italiano delle soluzioni alternative all'uso del congiuntivo 
Entrambe le lingue danno importanza al presente, usano in misura minore il condizionale, e più raramente il futuro, l'imperativo e il modo nominale. Il presente dell'indicativo è un'alternativa importante in entrambe le lingue: il testo a fumetti tenta di riprodurre il discorso orale dove l'uso del presente è quasi egemonico.

D'altra parte, le due lingue divergono sull'uso dell'indicativo imperfetto o più che perfetto, una soluzione maggioritaria in francese (40\%) e una soluzione minoritaria in italiano $(4,2 \%)$ : questa differenza si spiega con l'estensione in francese dell'ambito di utilizzo dell'indicativo imperfetto in seguito all'abbandono del congiuntivo imperfetto ${ }^{11}$. Sono state calcolate le soluzioni alternative in francese al congiuntivo imperfetto italiano. Su un totale di 78 voci (cioè il $34,2 \%$ del nostro corpus), l'indicativo imperfetto o più che perfetto rappresenta il $62 \%$ delle soluzioni: occorrenze molto più numerose del congiuntivo presente (16\%), dell'indicativo presente o passato prossimo (9\%), del condizionale $(8 \%)$, dell'infinito $(2,5 \%)$, del participio passato $(1,2 \%)$ o delle forme non verbali $(1,2 \%)$.

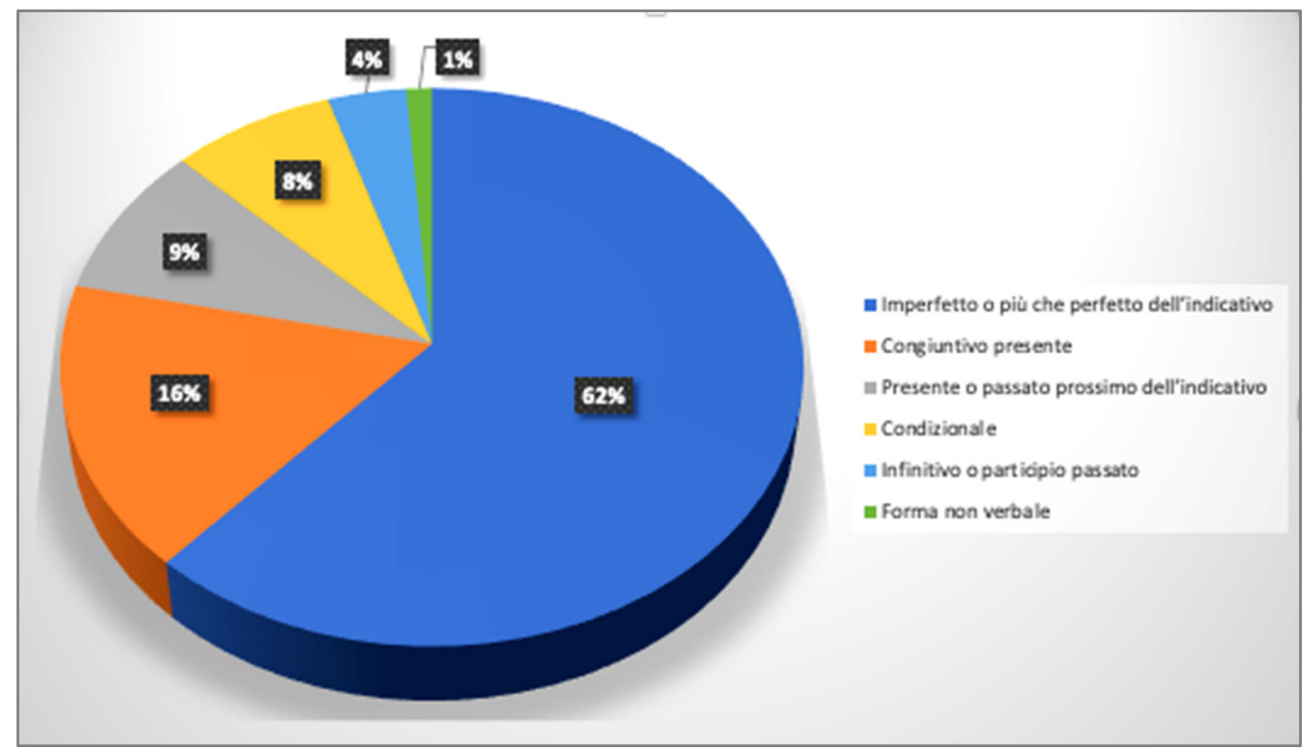

Figure 7. Soluzioni alternative in francese all'uso del congiuntivo imperfetto italiano

Il contesto di utilizzo dell'indicativo imperfetto o più che perfetto in francese equivalente ad un congiuntivo imperfetto o più che perfetto in italiano

11 (Cellard, 1996 : 7), (Blanche-Benveniste, 1997 : 53). 
è costituito principalmente da subordinate circostanziali di condizione (81\%) introdotte da "si", "si...et que", "comme si"; e in misura minore da completive e interrogative indirette (19\%). Si è trovato il congiuntivo presente soprattutto nelle completive $(77 \%)$ e l'indicativo presente per lo più in subordinate circostanziali di condizione (86\%). Si può constatare che, in generale, le completive richiamano il congiuntivo, mentre le subordinate circostanziali di condizione richiedono un tempo dell'indicativo. La nostra ipotesi è che in certe condizioni, in francese, l'informazione modale cede il passo all'informazione temporale e si usa l'indicativo piuttosto che il congiuntivo. Al contrario, quando l'informazione modale è necessaria, l'informazione temporale si perde perché c'è solo il presente al congiuntivo in francese, visto che l'imperfetto è scomparso da questo modo.

Nelle interrogative indirette, il verbo della reggente (comprendre, se demander) porta in seno alla sua semantica una virtualità sufficiente a far sì che il congiuntivo non sia necessario.

(Giov-fr-204) - Oui, il les étudiait pour comprendre d'où venait_l'argent.

(Giov-it-cond-51) - Sì, li studiava per capire da dove provenissero i soldi.

In alcune completive, il verbo della reggente (penser, croire, on aurait dit que, ignorer, ne pas savoir) porta la carica di virtualità e si usa l'indicativo. Per esempio, nello stesso traduttore (quello di Rencontre avec Pasolini), si rileva l'uso del congiuntivo presente nelle completive equivalente a un congiuntivo imperfetto nella versione italiana, principalmente quando il verbo della reggente è vouloir. In qualche occorrenza, il verbo della reggente è craindre o l'espressione il est fort probable que. Tuttavia, non troviamo gli stessi verbi di quelli con la subordinata completiva all'indicativo.

(Paso-fr-cond-14-b et c) - [...] Le voudrais qu'il m'appelle. Qu'il me fixe un nouveau rendez-vous. Je voudrais entendre à nouveau sa voix.

(Paso-it-cond-19-b et c) - [...] Vorrei che mi chiamasse, che mi desse un altro appuntamento, vorrei sentire ancore la sua voce, eccolo.

(Paso-fr-cond-9-c et d) - [...] On ne voulait pas que les Italiens parlent en dialecte. On ne voulait pratiquement pas qu'il y ait des paysans et des ouvriers.

(Paso-it-cond-11-c et d) - [...] Non si voleva che gli italiani parlassero dialetto, praticamente non si voleva che ci fossero dei contadini e degli operai.

vs.

(Paso-fr-212) - [...] J'ignorais ce qu'étaient ces objectifs.

(Paso-it-cond-40) - [...] e io non sapevo che cosa fossero questi obiettivi.

(Paso-fr-211) - [...] En fait, je pense qu'il récitait des poèmes...

(Paso-it-cond-49) - [...] Anzi penso recitasse poesie... 
Allo stesso modo, nell'autore di Goldy, l'unica occorrenza in francese di completiva con un congiuntivo tradotto da un congiuntivo imperfetto in italiano non ha lo stesso verbo nella reggente che le completive con l'indicativo:

(Goldy-fr-cond-5-b) - Dans ce cas, il vaudrait peut-être mieux que je prenne les commandes, vous ne croyez pas?

(Goldy-it-cond-5-b) - In questo caso forse sarebbe meglio che prendessi io i comandi, non crede?

vs.

(Goldy-fr-204) - J'ai vraiment cru que vous étiez le chacal !

(Goldy-it-cond-30) - Ho creduto davvero che Lei fosse lo sciacallo!

(Goldy-fr-203) - Vous pensiez que j'étais mort dans l'explosion!

(Goldy-it-cond-35) - Pensavi che fossi morto nell'esplosione!

Il contesto d'uso del condizionale in francese equivalente a un congiuntivo imperfetto o più che perfetto in italiano è vario (relativa, circostanziale di condizione, completiva), non sembra essere decisivo: il valore di base sembra piuttosto il rifiuto, da parte del locutore, di assumere la responsabilità del proprio discorso (Abouda, 2001).

Le due lingue si differenziano anche per l'uso dell'infinito e il ricorso a forme non verbali, soluzioni ricorrenti in italiano ma poco utilizzate in francese (infinito: il $29,7 \%$ in italiano contro il 3,4\% in francese; forme non verbali: il $23,4 \%$ in italiano contro il $6 \%$ in francese). L'italiano sembra favorire l'uso dell'infinito quando condivide lo stesso soggetto del verbo della reggente. E va sottolineato che per la metà delle occorrenze con l'uso dell'infinito il verbo della reggente è dovere.

\section{Conclusione}

Nel 50\% dei casi osservati nel nostro corpus, l'uso del congiuntivo in italiano corrisponde a soluzioni alternative in francese. Queste sono principalmente:

- Indicativo imperfetto o più che perfetto (42\%)

- Indicativo presente o il passato prossimo (37\%)

- Condizionale (7\%)

Abbiamo trovato nelle traduzioni che in francese le soluzioni alternative all'uso del congiuntivo imperfetto in italiano sono l'uso di: 
- Indicativo imperfetto o più che perfetto $(62 \%)$

- Congiuntivo presente (16\%)

- Indicativo presente o passato prossimo (9\%)

- Condizionale (8\%)

Si mantiene così la gerarchia delle soluzioni alternative al congiuntivo: l'indicativo imperfetto o più che perfetto è la soluzione maggioritaria, prima dell'indicativo presente o passato prossimo. Il francese non applica una concordanza dei tempi sistematica perché il più delle volte la carica della virtualità viene portata dalla semantica del verbo della reggente. Quando l'informazione modale non è più portata dal verbo della subordinata, l'informazione temporale (presente, passato) e quella aspettuale (compiuto-incompiuto) determinano la scelta di uno dei tempi dell'indicativo. L'uso del condizionale indica che il locutore non assume l'enunciato. I pochi casi in cui il francese usa il congiuntivo presente nella subordinata sono quelli in cui il verbo della reggente è vouloir, falloir, préférer, o le locuzioni verbali valoir mieux, être probable, juste, inutile, impossible, incroyable ecc., ma anche i verbi espérer, imaginer, croire che possono essere seguiti dall'indicativo o dal congiuntivo a seconda del grado di realtà o virtualità assegnato al processo della subordinata.

Nel $21 \%$ dei casi osservati nel nostro corpus, l'uso del congiuntivo in francese corrisponde a soluzioni alternative in italiano. Queste sono principalmente:

- Indicativo presente (32\%)

- Infinito (29\%)

- Forma non verbale $(23 \%)$

- Condizionale $(6 \%)$

Tuttavia, rimane un terzo dei casi osservati nel nostro corpus per i quali sia il francese che l'italiano usano il congiuntivo. Ma quando l'italiano applica la concordanza dei tempi tra la reggente e la subordinata, il francese non lo fa, avendo eliminato il congiuntivo imperfetto dalla sua cronogenesi. In francese, alcuni verbi della reggente richiedono il congiuntivo nella subordinata: si tratta di verbi la cui semantica non indica chiaramente la virtualità del processo che seguirà nella subordinata (vouloir, falloir ecc.). 


\section{BIBLIOGRAPHIE}

Abouda L. (2001), « Les emplois journalistique, polémique et atténuatif du conditionnel. Un traitement unitaire », Le conditionnel en français, Recherches linguistiques, Klincksieck, p. 277-294.

Begioni L., Rocchetti A. (2013), «Comprendre la concordance des temps et son évolution comme un phénomène de déflexivité : d'une concordance, élément actif de la syntaxe (italien, français classique) à une concordance en cours de réduction (français d'aujourd'hui) ", Langages, 2013/3, n¹91, p. 23-36.

Blanche-Benveniste C. (1997), Approches de la langue parlée en français, Gap/Paris, Ophrys. Brault-Scappini S.A. (2017), «Étude du dispositif d'extraction en C'EST...QU, différenciation entre une relative en C'EST...QU et une proposition clivée» in L'information grammaticale, $\mathrm{n}^{\circ} 114$, juin 2007, pp. 53-56.

Brunot F. (1965), La pensée et la langue, Paris, Masson.

Cellard J. (1996), Le subjonctif : comment l'écrire? quand l'employer ?, Paris, Duculot.

Chiusaroli F., Zanzotto F. M. (a c. di) (2012a), Scritture brevi di oggi, Napoli, Quaderni di Linguistica Zero 1. Sul sito: http://www.unior.it/userfiles/workarea_477 /Chiusaroli\%20e\%20Zanzotto\%20-\%20Scritture\%20Brevi\%2 0di\%200ggi.pdf

Chiusaroli F., Zanzotto F. M. (a c. di) (2012b), Scritture brevi nelle lingue moderne, Napoli, Quaderni di Linguistica Zero 2. Sul sito: http://www.unior.it/ userfiles/workarea_477/Scritture\%20Brevi\%20nelle\%20lingue\%20moderne $\% 20 \mathrm{a} \%$ 20cura\%20di\%20Chiusaroli\%20e\%20Zanzotto\%20\%20QUADERNI\%20DI\%20LZ\%202012.pdf

Colella G. (2012), "Modalità", Sintassi dell'italiano antico. La prosa del Duecento e del Trecento, a cura di Maurizio Dardano, Roma, Carocci, pp. 55-68.

Dardano M. (2009), Nuovo Manualetto di linguistica italiana, Bologna, Zanichelli, $5^{\mathrm{a}}$ ed.

Guide de grammaire française pour étudiants finnophones, § Les phrases clivées et pseudo-clivées, site en ligne :

http://research.jyu.fi/grfle/relatives-clivees.html, consulté le 14/08/2020.

Guillaume G. (1929 [1965]), Temps et Verbe. Théorie des aspects, des modes et des temps, suivi de L'Architectonique du temps dans les langues classiques, Paris, Champion. Traduzione italiana di A. Manco, Tempo e verbo. Teoria degli aspetti, dei modi e dei tempi, Università degli studi di Napoli "L'Orientale", Dipartimento di studi del mondo classico e del Mediterraneo antico, 2006.

Rouquier M. (2018), "Les constructions clivées », Encyclopédie Grammaticale du Français, en ligne : http://encyclogram.fr., consulté le 14/08/2020

Saffi S. (2014), « De l'intérêt d'une étude contrastive des bandes dessinées Topolino et Le journal de Mickey», Studia Universitatis Babeş-Bolyai Philologia, 2014/3, p. 7-23.

Saffi S. (2016), “Topolino e Le Journal de Mickey (anni Trenta): osservazione dei dimostrativi e avverbi di luogo afferenti", Quaderni di AION, Università degli Studi de Napoli L'Orientale, Alberto Manco e Azzurra Mancini (a cura di) Scritture brevi: segni, testi e contesti. Dalle iscrizioni antiche ai tweet, 5, p. 425-444. 
Squartini M. (2002), "Futuro e Condizionale nel discorso riportato", La parola al testo. Scritti per Bice Mortara Garavelli, a cura di Gian Luigi Beccaria e Carla Marello, Alessandria, Edizioni dell'Orso, pp. 451-462.

Squartini M. (2010), "L'espressione della modalità", in Salvi G., Renzi L., Grammatica dell'italiano antico, Bologna, Il Mulino, pp. 583-590.

Tekavčić P. (1972), Grammatica storica dell'italiano, 3 volumes: Fonematica, Morfosintassi, Lessico, Bologna, Il Mulino.

Wilmet M. (1997), Grammaire critique du français, Paris/Louvain-la-Neuve, Hachette/Duculot.

\section{Bibliographie secondaire}

Baru, Pelot, P. (2008), Pauvres Zhéros, Paris, Rivages/Casterman/Noir; Povere nullità, traduzione di Stefano Sacchitella, Bologna, Coconino Press, Fandango, 2010.

Blutch (1997), Peplum, Bordeaux, Éditions Cornélius; Peplum, traduzione italiana di Chiara Rea, Torino, 001 Edizioni, 2017.

BonaCCorso, L., Rizzo, M. (2009), Peppino Impastato, un giullare contro la mafia, Padova, Edizioni BeccoGiallo; traduzione francese, Mafia Tabloïds. L'histoire vraie d'un journaliste face à la Cosa Nostra, traduttore non specificato, Belgique, Ankama Éditions, 2011.

Catel (2008), Quatuor, fumetto adattato dai testi di Jacques Gamblin, José-Louis Bocquet, Thierry Bellefroid e Pascal Quignard, Paris, Casterman; Quartetto, traduzione italiana di Giliola Viglietti, Torino, 001 Edizioni, 2010.

Fonteneau, P., N'Galle Edimo, C., Masioni, P. (2005), L'Appel, Sasso Marconi, Edizioni LaiMomo; traduzione italiana, traduttore non specificato, L'Appello, stesso anno, stesso editore.

PeCqueur, D. (2002), Golden city, tome 4. Goldy, Paris, Guy Delcourt Production; Golden city, tome 4. Goldy, traduzione e adattamento dell'edizione italiana di Sergio Rossi e Laura Ridoni, Scandiano (RE), Edizioni BD, 2004.

StASSI, C., Di GREGorio, G. (2006), Brancaccio, storie di mafia ordinaria, Padova, Edizioni BeccoGiallo; traduzione francese di Laurent Lombard, Brancaccio. Chronique d'une mafia ordinaire, Paris, Casterman, 2007.

StaSSI, C., Garlando, L. (2008), Per questo mi chiamo Giovanni, Milano, Biblioteca Universale Rizzoli, C'est pour ça que je m'appelle Giovanni, traduzione francese di Claudia Migliaccio, Paris, Dargaud Bénélux, 2011.

Toffolo, D. (2002), Intervista a Pasolini, Pordenone, Edizioni Biblioteca dell'Immagine, Pasolini une rencontre, traduzione francese di Émilie Saada, Bruxelles, Casterman, collection Écritures, 2004. 